\title{
Parental feeding practices and child's level of food fussiness and neophobia - Preliminary analysis from NCFSII
}

\author{
Stephanie Rahill ${ }^{1}$, Aileen Kennedy ${ }^{1}$, Laura Kehoe ${ }^{2}$, Janette Walton ${ }^{2}$, Albert Flynn ${ }^{2}$, \\ Maria Buffini ${ }^{3}$, Breige McNulty ${ }^{3}$, Anne Nugent ${ }^{4}$ and John Kearney \\ ${ }^{1}$ Technological University of Dublin, Dublin, Ireland, \\ ${ }^{2}$ University College Cork, Cork, Ireland, \\ ${ }^{3}$ University College Dublin, Dublin, Ireland and \\ ${ }^{4}$ Queens University Belfast, Belfast, United Kingdom
}

\section{Abstract}

Research suggests that food fussiness (FF) and food neophobia (FN) are two separate constructs. Food fussiness is the tendency to be selective about a large proportion of familiar and unfamiliar foods, while food neophobia is the refusal of novel foods. Therefore, the aim of this study is to explore the association between parental feeding practices and child's FF or FN.

Analysis was based on cross-sectional data from the nationally representative Irish National Children's Food Survey II (NCFSII; 2017-2018). The NCFSII collected detailed eating behaviour data from children aged 5-12 $(\mathrm{n}=596)$ using the Children's Eating Behaviour Questionnaire (CEBQ). This questionnaire contained four items from the food fussiness subscale that represented FN and two items that represented FF. The Feeding Practices \& Structure Questionnaire (FPSQ) assessed non-responsive and structurerelated parental feeding practices. Spearman's correlation established the association between parental feeding practices and child's FF and FN. Moderation analyses was conducted to explore the extent to which child's age moderates the association.

Higher levels of child's FN was weakly to moderately associated with higher parental reports of reward for eating $(\mathrm{RE})(r=.210$, $\mathrm{p}<0.001)$, persuasive feeding (PF) $(r=.340, \mathrm{p}<0.001)$, overt restriction (OR) $(r=.195, \mathrm{p}<0.001)$ and lower reports of structured meal settings (SMS) $(r=-.085, \mathrm{p}=0.039)$ and family meals (FMS) $(r=-.387, \mathrm{p}<0.001)$. Higher levels of child's FF had a slightly stronger association with the same parental feeding as FN, with additional associations with structured meal timings (SMT) ( $r$ $=-.089, \mathrm{p}=0.031)$ and covert restriction $(\mathrm{CR})(r=-.083, \mathrm{p}=0.045)$. Age moderated the association between both child's FF $[b$ $=.22, \mathrm{p}<0.001]$ and $\mathrm{FN}[b=.17, \mathrm{p}=0.002]$ and parental reports of PF, along with moderating the association between child's $\mathrm{FF}[b=.11, \mathrm{p}=0.04]$ and parental reports of SMT.

Overall, child's FN and FF were both associated with higher levels of non-responsive feeding practices and lower reports of structure-related feeding practices, with child's FF associated with more parental feeding practices than FN. These findings suggest that it is important to address FN and FF as separate constructs, with more structure-related feeding practices inversely associated with higher levels of FF only. In addition, as children get older findings suggest that less parental feeding practices are utilised, however, higher levels of child's FF/FN are associated with similar levels of PF and SMT (FF only) regardless of age.

\section{Conflict of Interest}

"There is no conflict of interest". 